\title{
Gesellschaftliche Konsequenzen internationaler Abkommen zur Alterspolitik
}

Wenngleich der demografische Wandel unzweifelhaft massive gesellschaftliche Umwälzungen mit sich bringt, werden die damit verbundenen Handlungsnotwendigkeiten noch immer unterschätzt. Möglicherweise genießt deshalb die Altenpolitik im Vergleich zu anderen Politikfeldern auch heute noch kein besonders hohes Ansehen. Bei Umfragen in Deutschland ist nur den wenigsten Bürgern überhaupt die Existenz des Ministeriums bekannt, das sich mit Senioren befasst. Die Altenpolitik gilt vielmehr als Nischenbereich ohne breiten politischen Rückhalt und ohne die Möglichkeit, sich auf dem politischen Parkett medienwirksam zu profilieren (vgl. Pohlmann, 2003). Noch fehlt ein grundlegendes Verständnis dafür, dass das wachsende Ungleichgewicht zwischen Jung und Alt in der Bevölkerung unsere Politik entscheidend prägen muss. Trotz der offensichtlichen Bevölkerungsentwicklung und in Anbetracht der nur zögerlichen Berücksichtigung dieses Prozesses ist auf diesem Gebiet daher nach wie vor besondere Überzeugungsarbeit gefordert.

Weitgehend unbemerkt von der medialen Berichterstattung und damit gleichzeitig auch ohne nennenswerte Spuren in der öffentlichen Auseinandersetzung hat sich aber doch zumindest in der internationalen Altenpolitik eine deutliche Trendwende vollzogen. Klares Indiz dafür sind die jüngst zu beobachtenden Entwicklungen bei den Vereinten Nationen. Angesichts der weltweit zu erwartenden Verschiebungen der Altersstrukturen sah sich der Staatenbund veranlasst, eine gemeinsame politische Offensive zu starten. Nach fast zwei Jahrzehnten des politischen Stillstands auf diesem Gebiet konnte im April 2002 ein neuer Weltaltenplan (auch Madridplan genannt, vgl. United Nations, 2002) verabschiedet werden, der diese Bemühungen deutlich macht. 191 Mitgliedstaaten haben sich in dem Dokument über Ziele und Lösungswege verständigt. Gleichzeitig wurden Absichtserklärungen abgegeben, wie die Staaten mit den Folgen einer langfristigen Alterung der Bevölkerung zukünftig umzugehen beabsichtigen.

Verständlicherweise bleiben aufgrund der unterschiedlichen Ausgangsituationen in den verschiedenen Staaten die Thesen dieser zentralen Konvention entweder vage oder an konkreten Punkten ohne völkerrechtliche Verbindlichkeit. Schon 1982 ist genau aus diesem Grund ein vorangegangener und ebenso ehrgeiziger Ansatz gescheitert (vgl. United Nations, 1982). Obwohl bereits damals die globale Bevölkerungsentwicklung richtig interpretiert und wesentliche Handlungsoptionen aufgezeigt worden waren, ist es in der Folgezeit nicht gelungen, diese Vorschläge aufzugreifen und grenzüberschreitende Maßnahmen zu ergreifen. Bemerkenswert erscheint vor diesem Hintergrund der aktuelle Versuch, eine regionale Konkretisierung des neuen Weltaltenplans zu veranlassen. Auf besondere Initiative der bundesdeutschen Regierung wurde unter der Federführung der zuständigen Untergliederung der Vereinten Nationen, der sogenannten Wirtschaftskommission für Europa (Economic commission for Europe, UNECE), bereits wenige Monate später ein Nachfolgedokument von den 
beteiligten Mitgliedstaaten verabschiedet (vgl. UNECE, 2002). Die Besonderheit dieser als Berlinplan bezeichneten Implementierungsstrategie liegt in dem Bemühen, möglichst klare Verbindlichkeiten zu formulieren. Die weiteren Ausführungen in diesem Beitrag gehen auf die erste der dort nachzulesenden Verpflichtungen ein. ${ }^{1}$ Die Staaten vereinbaren an dieser Stelle, die Dimension des Alterns in alle politischen Bereiche unmittelbar einzubeziehen.

$\mathrm{Zu}$ Recht stellen die Mitgliedsländer die Bedeutung eines Querschnittsansatzes an den Anfang des Dokuments. Die Umsetzung eines derart ganzheitlichen Ansatzes ist jedoch in der Praxis mit vielfachen Schwierigkeiten und Hürden verbunden. Das zeigt sich auch in den Resolutionen der Vereinten Nationen. So wird man beispielsweise vergeblich in den Dokumenten des im Mai 2002 in New York durchgeführten Weltkindergipfels (United Nations, 2002b) nach einem Hinweis auf ältere Menschen und die Wechselwirkungsprozesse des demografischen Wandels für unterschiedliche Altersgruppen suchen. Nur einen Monat nach der Weltversammlung zu Altersfragen muss eine solche fehlende Anbindung verwundern. Mangelnde inhaltliche Vernetzungen wie diese sind leider noch immer eher die Regel als die Ausnahme. Umso notwendiger erscheint es, die unterschiedlichen Facetten für einen Querschnittsansatz genauer zu beleuchten. Leider fehlt in der regionalen Implementierungsstrategie der UNECE eine Ausdifferenzierung dieser ganz wesentlichen Querschnittspolitik.

Deshalb sollen im Weiteren einige wichtige Aspekte eines breiten demografischen Ansatzes herausgegriffen und im Hinblick auf seine Bedeutung für die zukünftige Bevölkerungsentwicklung hin analysiert werden. Diese Beispiele verdeutlichen, dass das Altern nicht nur für den Einzelnen, sondern auch für die Gesellschaft eine stetig wachsende Bedeutung hat. Der demografische Wandel wird hierbei als ein System verstanden, das eine integrative und koordinierte Herangehensweise verschiedener relevanter Aspekte dringend erforderlich macht. Will man auf die historisch einmaligen Entwicklungen und die damit verbundenen epochalen Konsequenzen angemessen antworten, so ist eine Reihe von Mindestanforderungen zu berücksichtigen. Die weiteren Ausführungen gehen auf diese Anforderungen schlaglichtartig ein und belegen, dass erst eine breite Berücksichtigung von Altersfragen dazu beitragen kann, die zentralen gesellschaftlichen Herausforderungen der Zukunft wirksam zu meistern.

\section{Auswirkungen des Alterns differenzieren}

Es liegt auf der Hand, dass die Verschiebungen der Altersanteile in der Bevölkerung die unterschiedlichsten Themenfelder betreffen. Der demografische Wandel hat hierbei sprichwörtlich revolutionäre Auswirkungen vor allem auf die Bereiche Wirtschaft, Soziales, Arbeit und Gesellschaft. Durch die aktuelle und zukünftige Bevölkerungsentwicklung entstehen einerseits große Potenziale, andererseits stellen sich enorme

1 Bei den nachfolgenden Überlegungen handelt es sich um eine gekürzte und aktualisierte Fassung eines bereits erschienenen Artikels des Autors. Das Original trägt den Titel »Erfordernisse einer gesellschaftlichen Querschnittspolitik « und ist 2003 in dem von Stefan Pohlmann herausgegebenen Sammelband »Der demografische Imperativ« erschienen. 
Herausforderungen. Seit geraumer Zeit schenken Presse, Funk und Fernsehen diesen neuen Herausforderungen zunehmende Beachtung. Es fehlt jedoch oftmals eine übergreifende Perspektive. Betrachtet man die vielfältigen und miteinander in Wechselwirkung stehenden Anforderungen und Aufgabenbereiche, die der demografische Wandel für Staat und Gesellschaft mit sich bringt, so wird deutlich, dass singuläre Ansätze per se zum Scheitern verurteilt sein müssen. Vielmehr sind Herangehensweisen erforderlich, die in der Psychologie als komplexe Problemlösestrategien bezeichnet werden.

Dörner (vgl. Dörner, Kreuzig, Reither, Stäudel, 1995) hat anschaulich gezeigt, unter welchen Voraussetzungen Handlungsansätze optimiert werden können. Die komplexe Dynamik unterschiedlicher Wirkungsfaktoren kann dabei mitunter zu unerwarteten Effekten führen und macht insofern eine sensible Überwachung der Prozesse sowie rechtzeitige Kurskorrekturen erforderlich. Nur wenn es gelingt, Mechanismen und Störvariablen zu erkennen und ihre Wirkungsweise langfristig zu überprüfen, ist mit zufriedenstellenden Ergebnissen zu rechnen. Diese Analogie trifft auch unmittelbar auf die Herausforderungen des demografischen Wandels zu. Allerdings ist in der Realität die Anzahl der beteiligten Variablen natürlich um ein Vielfaches höher als in einem kognitiven Simulationsprojekt.

In vielen Beiträgen, die den Versuch unternehmen, zentrale Themen in Bezug auf den demografischen Wandel zu benennen, herrscht eine primär monetäre und problemorientierte Sichtweise vor. Die Themen Gesundheit und soziale Sicherung nehmen dabei Spitzenpositionen in der öffentlichen Diskussion ein. Eine Auseinandersetzung mit diesen Fragen ist dringend nötig. Niemand wird zudem heute in Abrede stellen wollen, dass ausschließlich umlageorientierte Systeme in einer alternden Gesellschaft keinen Bestand haben können und neue Finanzierungsmodelle zwingend erforderlich sind. Allein die Zunahme älterer Menschen impliziert aber keine zwangsläufige Kostenexplosion. Eine kritische Einstellung ist berechtigt, darf aber nicht dazu führen, existierende Handlungsoptionen zu vernachlässigen. Ausschließlich negative Zukunftsszenarien erscheinen demgegenüber nicht nur fatalistisch, sondern wirken auch im höchsten Maße destruktiv. So sind heute beispielsweise die Bereiche Prävention und Rehabilitation noch deutlich ausbaufähig und können lang- und mittelfristig zu erheblichen Einspareffekten führen. Diese Sichtweise findet sich auch in dem Bericht der Enquête Kommission Demographischer Wandel (Deutscher Bundestag, 2002). Ältere Menschen sind nicht als Problem, sondern vielmehr als Teil der Lösung zu betrachten. Nur auf diese Weise lässt sich die vielfach zu beobachtende Konzeptlosigkeit und Handlungsparalyse zum Thema Altern aufbrechen.

Wir brauchen demnach einen realistischen Ansatz, der Risiken und Chancen klar herausarbeitet, Probleme nicht verschweigt, aber dennoch Raum und Mut für neue Konzepte lässt. Wer lediglich gängige Klischees bedient, wird der Breitenwirkung des Alterns mit seinen gestaltbaren Folgen nicht gerecht.

\section{Herausforderung für alle Generationen erkennen}

Altenpolitische Entscheidungen müssen dahingehend überprüft werden, welche Auswirkungen sie auf andere Altersgruppen haben. Maßnahmen für Senioren richten 
sich nicht zwangsläufig gegen jüngere Menschen. Ein generationenübergreifender Handlungsansatz ist vielmehr dem Motto der Vereinten Nationen verpflichtet, eine Gesellschaft für alle Generationen zu schaffen oder aufrecht zu erhalten. Dieses Leitmotiv ist mit dem Slogan »towards a society for all ages « bei den Vereinten Nationen bereits seit geraumer Zeit berücksichtigt. Es geht auf den 1995 durchgeführten Sozialgipfel in Kopenhagen zurück und hat auch 1999 für das Internationale Jahr der Senioren als wesentliche Orientierung gedient. Der Bericht der UNO-Generalversammlung mit dem Titel »Social development, including questions relating to the world social situation and to the youth, ageing, disabled persons and the family « hat die Notwendigkeit eines intergenerativen Verständnisses deutlich gemacht. In dem Papier heißt es:

»Das Älterwerden ist zweifellos ein multidimensionales, multisektorielles und multigeneratives Problem. Es umfasst die Situation der älteren Menschen, die lebenslange Entwicklung des einzelnen, die Beziehungen zwischen den Generationen und die Beziehung zwischen dem Alterungs- und dem Entwicklungsprozess der Gesellschaft. [...] Eine Gesellschaft wird als eine umfassende Gesellschaft gesehen, die auf der Achtung aller Menschenrechte und Grundfreiheiten, der kulturellen und religiösen Vielfalt, der sozialen Gerechtigkeit, der demokratischen Mitwirkung und der Rechtsstaatlichkeit beruht. So können wir uns eine Gesellschaft für alle vorstellen als eine Gesellschaft, die ihre Strukturen und Funktionsweise wie auch ihre politischen Konzeptionen und Pläne den Bedürfnissen und Fähigkeiten aller anpasst und so die potenziellen Kräfte aller zum Wohle aller freisetzt. Eine Gesellschaft für alle Altersgruppen würde darüber hinaus die Generationen dazu befähigen, gegenseitig ineinander zu investieren und die Früchte dieser Investitionen zu genießen, geleitet von den sich ergänzenden Grundsätzen der Gegenseitigkeit und der Gleichbehandlung." (Bericht A/50/114vom 22. März. 1995; Paragraphen 36 und 38)

Alarmierende Berichte über ein drohendes Zerwürfnis der Generationen und einen wachsenden Mangel an Solidarität häufen sich. Aus Pressebeiträgen und Sendungen in Funk und Fernsehen entsteht hierbei bisweilen der Eindruck eines bereits seit geraumer Zeit andauernden »Krieges« zwischen Jung und Alt (vgl. Bosch, 1990). Auch in einer Reihe von Fachpublikationen finden sich außerordentlich pessimistische Hinweise über das Generationenverhältnis (z. B.: Gronemeyer, 1991; Mohl, 1993; Schüller, 1995). Betrachtet man die existierenden wissenschaftlichen Untersuchungen auf diesem Feld allerdings eingehender, zeigt sich, dass Katastrophenmeldungen über den Zerfall der Familie oder permanente Konflikte zwischen den Generationen zumindest gegenwärtig in Deutschland unbegründet sind (Wagner et al., 1996; Kohli et al., 2000). Unsere Gesellschaft ist belastbarer als vielfach angenommen, und das Prinzip der Gegenseitigkeit wird auch heute noch aktiv gelebt. Die Transferleistungen sowohl materieller als auch immaterieller Art zwischen Jung und Alt sind dabei erheblich.

Auch trotz oder manchmal vielleicht sogar wegen einer räumlichen Distanz zwischen erwachsenen Mitgliedern einer Familie lassen sich keine Indizien für ein gestörtes Verhältnis zwischen den Generationen ausmachen. Vielmehr werden Generationenbeziehungen durch die Formel »Innere Nähe bei äußerer Distanz« anschaulich beschrieben. Der sich abzeichnende Strukturwandel in der Familie, der zu einem 
raschen Anwachsen von Kleinfamilien, Einelternfamilien, Patchworkfamilien und Singlehaushalten geführt hat, stellt die Familie aber gerade in Zukunft vor erhebliche Belastungen. Natürlich gibt es - und das ist kein neues Phänomen unserer Zeit - Konfliktbereiche zwischen den Generationen. Die Konflikte bestehen aber in der Regel nicht unmittelbar zwischen Großeltern und Enkeln.

Zusammenfassend bleibt festzustellen, dass Negativschlagzeilen über die fehlende Generationensolidarität den Generationenkrieg eher herbeireden als beschreiben. Problematisch erscheint allerdings auch aus heutiger Sicht, dass der Kontakt zwischen den Generationen außerhalb der Familie stark eingeschränkt ist und dass dieser fehlende Austausch vielfach Anlass für bestehende Missverständnisse zwischen den Generationen bietet. Vor allem deshalb sind Initiativen zu begrüßen, die das Miteinander von Jung und Alt fördern. Auch der Begriff der Familie bedarf aus heutiger Sicht einer weiteren definitorischen Klärung und sollte über tradierte Familienbilder hinausgehen.

Außerdem ist dafür Sorge zu tragen, dass Altersdiskriminierungen abgebaut und den bestehenden Richtlinien des Amsterdamer Vertrages (vgl. Presse- und Informationsamt der Bundesregierung, 1999) auch auf nationaler Ebene zur Geltung verholfen wird. Der am 1. Mai 1999 in Kraft getretene Vertrag ist das Ergebnis der 1996 in Turin durchgeführten Regierungskonferenz und hat das Ziel, die Europäische Union bürgernäher zu gestalten und ihre politische Identität nach innen und außen sichtbarer und wirksamer werden zu lassen. Bislang zeigen sich jedoch in den Mitgliedsländern noch immer offene oder verdeckte Formen der Ausgrenzung verschiedener Altersgruppen (vgl. Büro gegen Altersdiskriminierung, 2002). Nach einer EurobarometerUmfrage stellt das Alter gefolgt von der ethnischen Herkunft die mit Abstand häufigste Form der selbst erfahrenen und beobachteten Ungleichbehandlung dar (vgl. EUNachrichten, 2004).

Generationsübergreifende Ansätze müssen demnach innerhalb und außerhalb der Familie bestehen. Rahmenstrukturen, die die Solidarleistungen zwischen den Generationen fördern und die Ausgrenzung einzelner Altersgruppen aktiv verhindern, sind hierbei in Zukunft weiter auszubauen oder auch neu zu etablieren. Die Motivation für entsprechende gesellschaftspolitische Schritte beruht auf der wachsenden Erkenntnis, dass durch ein Miteinander von Jung und Alt gemeinsame Interessen gewahrt werden.

\section{Maßnahmen auf Nachhaltigkeit prüfen}

Die Berücksichtigung der Lebensspanne ist gerade in der Gerontologie zu einer Grundforderung geworden (vgl. Thomae, 1983). Sie macht deutlich, dass alte Menschen eine Biografie mitbringen, die sie einzigartig macht. Maßnahmen müssen sich an dieser individuellen Lebensgeschichte orientieren. Ein nachhaltiger Handlungsansatz sollte diese Erkenntnis beherzigen und sowohl die Vergangenheit als auch die sich davon ableitenden Gestaltungsspielräume der Zukunft prüfen. Politisches Vorgehen erfordert dabei nicht nur Sorgfalt und eine gezielte Recherche, sondern auch den Mut zu Visionen und unpopulären Entscheidungen. 
Vielfach wird der Politik vorgeworfen, dass sie langfristig zu erwartende Negativfolgen zugunsten kurzfristiger positiver Effekte billigend in Kauf nimmt. Eine Querschnittspolitik durchbricht derartige Mechanismen und setzt kurzsichtigen Strategien das Prinzip der Nachhaltigkeit entgegen. Insbesondere altenpolitische Entscheidungen dürfen sich nicht allein auf das Hier und Jetzt konzentrieren. Vielmehr müssen sie prospektiv angelegt sein und die Konsequenzen für die Senioren von morgen und übermorgen kritisch bedenken. Nur so wird die oben geforderte Solidarität zwischen den Generationen auch politisch glaubwürdig; denn der Generationenvertrag setzt darauf, dass auch die zukünftigen Alten auf eine solidarische Gesellschaft vertrauen können. Konzentrieren sich politische Maßnahmen lediglich auf die Dauer von Legislaturperioden und wägen die Auswirkungen heutiger Entscheidungen auf die nächsten 10, 20 oder sogar 30 Jahre nicht angemessen ab, sind Probleme vorprogrammiert.

Da man in der Regel die Wirkungen aktueller Entscheidungen auf die zukünftige Entwicklung nicht vollständig absehen kann, ist gleichzeitig eine retrospektive Orientierung gefordert. Mit anderen Worten, wir müssen lernen, aus den Fehlern und Erfolgen der Vergangenheit zu lernen. Gleichzeitig ist die kontinuierliche Weiterverfolgung und Evaluation bereits eingeleiteter Schritte notwendig. So erweist sich rückblickend das zwei Jahrzehnte andauernde »Koma« des ursprünglich 1982 erstellten Weltaltenplans als fataler Fehler. Wichtige Empfehlungen und überfällige Aktionen wurden aufgrund dieser mangelnden Umsetzung in vielen Ländern nicht rechtzeitig eingeleitet. Die Folgekosten sind nach zwei Jahrzehnten damit erheblich und wären in weiten Teilen vermeidbar gewesen.

Unmittelbar vor der UNECE Ministerkonferenz fand in Johannesburg der Weltgipfel über nachhaltige Entwicklung statt (World Summit on Sustainable Development). Dieser Klimagipfel baut auf den Ergebnissen der UN-Umweltkonferenz aus dem Jahr 1992 in Rio de Janeiro auf, der mit der Verabschiedung der Agenda 21 die Weichenstellung für eine nachhaltige Entwicklung vorgenommen hat. Im Zentrum von Johannesburg stand ein weltweit wirksamer Klimaschutz und eine gesicherte Energieversorgung. Auch wenn viele internationale Umweltorganisationen von den Ergebnissen der Konferenz enttäuscht waren, so unterstreicht der Gipfel immerhin die Verantwortung für nachkommende Generationen. Die Erhaltung der Umwelt erfordert dabei ebenso nachhaltige Strategien wie auch der demografische Wandel. Bemerkenswert ist in diesem Zusammenhang, dass auf nationaler Ebene bereits im Vorfeld zu diesem Weltgipfel von den Koalitionsfraktionen eine nationale Nachhaltigkeitsstrategie angeregt wurde (vgl. Presse- und Informationsdienst der Bundesregierung, 2001). Diese Strategie sieht eine gleichrangige Berücksichtigung ökonomischer, ökologischer und sozialer Belange vor und konzentriert sich in Form eines sektorübergreifenden Ansatzes auf vier Kernthemen. Es geht dabei um
a) Generationengerechtigkeit,
b)Lebensqualität,
c) sozialen Zusammenhalt und
d)internationale Verantwortung.

Diese Leitlinien stehen auch in Anbetracht des demografischen Wandels im Fokus der Aufmerksamkeit. Auch hier muss also das Rad nicht neu erfunden werden. Auf- 
gabe muss es vielmehr sein, die Notwendigkeit für nachhaltige politische Entscheidungen immer und immer wieder anzumahnen.

\section{Globale Dimension des Alterns einbeziehen}

Der demografische Wandel bezeichnet ein nahezu weltweites Phänomen. Die Verschiebungen der Altersgrenzen machen nicht an nationalen Grenzen halt, sondern vollziehen sich auf allen Kontinenten. Allerdings bestehen in verschiedenen Ländern mitunter beträchtliche Unterschiede im Hinblick auf das Ausmaß und die Geschwindigkeit dieser demografischen Veränderungen. Gerade in Entwicklungsländern verschieben sich die Altersanteile in der Bevölkerung außerordentlich rasant. Im Vergleich zu einigen Industrienationen verläuft dieser demografische Wandel bis zu viermal so schnell (vgl. United Nations, 2002b). Diese dramatischen Prognosen führen in den Entwicklungsländern aufgrund ihrer in weiten Teilen rudimentären sozioökonomischen Versorgungskapazitäten zu einer Verschärfung sozialer Problemlagen.

Im internationalen Vergleich bestehen aber nicht nur Unterschiede zwischen ungleich entwickelten Ländern, sondern auch innerhalb einzelner Länderkategorien. So finden sich etwa in der dritten Welt deutliche Diskrepanzen zwischen einzelnen Staaten. Während beispielsweise Asien und Lateinamerika bis zum Jahr 2050 mit einem Anteil zwischen 20 und 25 Prozent vergleichsweise schnell altern, erreichen die von HIV/AIDS gravierend betroffenen afrikanischen Länder südlich der Sahara nur die Hälfte dieser Quote. Für die von der Aids-Pandemie besonders gegeißelten Regionen liegt die Lebenserwartung für das Jahr 2015 etwa fünf Jahre unterhalb der Grenze, die diese Länder ohne Aids erreichen würden. Wegen des anhaltenden Bevölkerungswachstums in genau diesen Ländern sagen die Projektionen der Vereinten Nationen aber selbst für ein Land wie Botswana mit einer HIV-Infektionsrate von 36 Prozent bis zum Jahre 2050 ein Bevölkerungswachstum von 37 Prozent vorher (UNFPA, 2001). Das nach wie vor bestehende globale Bevölkerungswachstum ist dabei auf die ärmeren Länder dieser Welt zurückzuführen. Es gilt die Faustregel, dass diejenigen Länder mit den höchsten Pro-Kopf-Einkommen die niedrigsten Geburtenraten aufweisen.

Angesichts dieser Überlegungen stellt sich die Frage, ob ein internationaler Ansatz für so heterogene Staaten überhaupt Sinn macht. Die Antwort muss lauten: Internationale Kooperation macht nicht nur Sinn, sondern ist schlicht zu einer unabdingbaren Notwendigkeit geworden. Stattdessen ist zu überlegen, worin die gemeinsamen Herausforderungen bestehen, die sich durch den demografischen Wandel weltweit stellen. Die Ziele und Handlungsnotwendigkeiten unterschiedlicher Länder fallen vielfach identisch aus. Globalisierung und Internationalisierung setzen die Gesellschaften unter steigenden Druck und erfordern neue Konzepte für den sozialen Zusammenhalt in nationaler und internationaler Hinsicht. Vielfach führt das zu spürbaren Einschränkungen des Spielraums nationaler Regierungen. Will man aber die oft beschworenen Synergieeffekte einer solchen Zusammenarbeit auch faktisch nutzen, bedarf es eines intensiven Austauschs verschiedener Nationen. Dabei bieten sich bestimmte Konstellationen der Länderzusammenarbeit möglicherweise eher an als andere. Aber ohne die Bereitschaft, eigene Erfolge und Misserfolge weiterzugeben, und ohne den festen Wil- 
len, aus den wertvollen Erfahrungen anderer Staaten $\mathrm{zu}$ lernen, wird man ein beschränktes und provinzielles Denken nicht überwinden können.

Woran es für eine internationale Herangehensweise nach wie vor mangelt, ist die Sammlung vergleichbarer Daten, die Entwicklung gemeinsamer Indikatoren und der Austausch von nachahmenswerten und erfolgreich erprobten Initiativen, Modellen und Programmen. Ebenso fehlt ein Austausch über Fehlschläge und Misserfolge, damit andere Länder vor vergleichbaren Fehlern bewahrt werden. Wir brauchen daher einen grenzüberschreitenden Ansatz, der nicht nur geografische Barrieren, sondern auch Grenzen im Kopf zu überwinden vermag.

\section{Eine vernetzte Gesellschaftspolitik verwirklichen}

Politische Entscheidungen werden auf unterschiedlichen Ebenen getroffen. Voraussetzung für einen politikübergreifenden Ansatz ist ein reibungsloses Zusammenspiel und ein wechselseitiger Informationstransfer zwischen diesen Ebenen. Dies gilt sowohl auf internationaler wie auch auf nationaler Ebene.

Im internationalen Bereich sind insbesondere die Prozesse innerhalb der Vereinten Nationen und der Europäischen Union von Bedeutung. Obwohl die verabschiedeten Resolutionen der Vereinten Nationen keiner einklagbaren Rechtsvorschrift entsprechen, werden auch auf dieser Ebene wichtige Kursentscheidungen getroffen. Die Erfahrungen zeigen allerdings, dass eine Reihe von Vereinbarungen der Vereinten Nationen auf nationaler Ebene nicht aufgegriffen wird. In vielen Fällen sind Sprachbarrieren die Ursache dafür. Außerdem sind UNO-Beschlüsse aufgrund der Flut von Dokumenten und der mangelnden Kodierung mit Schlüsselbegriffen mitunter schlecht auffindbar.

Anders verhält es sich mit Prozessen auf EU-Ebene. Entsprechende Vereinbarungen liegen in der Regel auch in deutscher Sprache vor und werden aufgrund ihrer unmittelbaren Implikationen für die nationale Gesetzgebung auch in Deutschland rezipiert. Obgleich altenpolitische Fragen nach dem Prinzip der Subsidiarität in die Verantwortlichkeit der einzelnen Mitgliedstaaten gestellt werden, finden sich doch im Amsterdamer Vertrag klare Vorgaben im Hinblick auf die Bekämpfung von Diskriminierung und sozialer Ausgrenzung im Alter. Dennoch steht eine nationale Umsetzung dieser Forderungen noch aus. Eine unklare Diktion und bestimmte juristische Vorbehaltsklauseln im Hinblick auf die Folgen dieser Vereinbarungen lassen sich als Erklärung für diesen Umstand ausmachen.

In Deutschland, das im Gegensatz zu vielen anderen Ländern über das Privileg einer eigenen Seniorenabteilung in einem Bundesministerium (Bundesministerium für Familie, Senioren, Frauen und Jugend) verfügt, werden notgedrungen nicht alle relevanten Aufgaben allein dort bearbeitet. Das neue Gesundheits- und Sozialministerium sowie das neue Wirtschafts- und Arbeitsministerium, aber auch andere Bundesbehörden nehmen jeweils eigenständige Kernkompetenzen für sich in Anspruch. Der demografische Wandel macht eine enge Zusammenarbeit und die Überwindung potenzieller Kompetenzstreitigkeiten zwischen diesen Instanzen unerlässlich. Möglicherweise kann man an dieser Stelle von den Erfahrungen anderer Nationen profitieren. So hat 
man in Australien ein übergreifendes Ministerium für Altersfragen etabliert, um auf die neuen Anforderungen des demografischen Wandels schneller reagieren zu können.

Ein politikübergreifender Ansatz erfordert in Deutschland eine starke Vernetzung zwischen Bund, Ländern und Gemeinden. Ziel muss es sein, politische Beschlüsse den Betroffenen auf lokaler Ebene spürbar zugute kommen zu lassen. Die Herausforderungen des demografischen Wandels dürfen nicht für einen politischen Schlagabtausch instrumentalisiert werden. Vielmehr ist auch über Fraktionen hinweg die Suche nach gemeinsamen Lösungen elementar. Dabei bleibt unbedeutend, durch welche Fraktion derartige Konzepte vorgeschlagen werden. Argumente - und nicht die Zugehörigkeit zu einer bestimmten Partei - sollten die erforderlichen politischen Entscheidungen motivieren. Die Vermeidung von Reibungsverlusten zwischen verschiedenen Ebenen, die Bündelung und Koordination von Kompetenzen in verschiedenen Ressorts, die Ausnutzung von Synergieeffekten und eine überparteiliche Koalition politischer Mandatsträger gehören insofern $\mathrm{zu}$ den wesentlichen Aufgaben unserer Politik. Solange aber strukturelle Hürden unterschiedlicher Ebenen nicht überwunden werden, lässt sich eine punktuelle Altenpolitik nicht überwinden (vgl. Dieck, 1991).

\section{Gesellschaftliche Kräfte mobilisieren}

Es wurde bereits auf die Vielschichtigkeit und Vielfältigkeit der demografischen Herausforderungen hingewiesen. Unbestritten muss sich die Politik diesen Aufgaben dringend stellen. Man kann aber bereits jetzt mit Bestimmtheit vorhersagen, dass staatliches Handeln allein nicht ausreichen wird, um dem massiven Wandel der Bevölkerungsentwicklung angemessen Rechnung zu tragen. Vielmehr ist die Identifikation weiterer Akteure zwingend. Es gilt hierbei, Bündnispartner für eine Kooperation zu gewinnen, Verantwortlichkeiten herauszuarbeiten und Handlungskompetenzen und Handlungsspielräume der Beteiligten auszumachen und gemeinsam zu nutzen.

Vorbildlich für den UNECE-Prozess war die Beteiligung der Wissenschaft und Zivilgesellschaft. In insgesamt drei Expertenseminaren wurde durch Wissenschaftler und Wissenschaftlerinnen unterschiedlicher ECE-Mitgliedsländer und die ausgewogene Einbeziehung verschiedener Fachdisziplinen der Grundstein für die regionale Implementierungsstrategie gelegt. Aufbauend auf dem wissenschaftlichen Input und im engen Austausch mit dem globalen Prozess der Überarbeitung des Weltaltenplans konnten auf dieser Basis die Textverhandlungen der Mitgliedsländer realisiert werden. Außerdem fand in allen Phasen der UNECE-Vorbereitung eine bislang nicht da gewesene Partizipation der Nichtregierungsorganisationen statt. Anders als für Regierungskonferenzen sonst üblich waren Vertreter der Nichtregierungsorganisationen nicht nur in den Expertensitzungen vertreten, sondern auch unmittelbar in den Textverhandlungen. Die Kommentierungen der Nichtregierungsorganisationen waren hierbei an vielen Stellen für die Mitgliedsländer außerordentlich hilfreich. Auch auf der Ministerkonferenz selbst waren nichtstaatliche Akteure mit eigenen Beiträgen beteiligt.

In einem frühen Textentwurf der regionalen Implementierungsstrategie, den die UNECE auch auf ihrer Website veröffentlicht hat, war ein ausführlicher Textabschnitt über die Einbeziehung der Wirtschaft, der Wissenschaft und der Zivilgesellschaft vor- 
gesehen. Unter der Überschrift »The Role and Responsibilities of different societal Actors in the Implementation of the Strategy: Principal Goals and Values « wies das Dokument der UNECE (2002b) auf das erforderliche Zusammenwirken von Regierungen, Zivilgesellschaft und Nichtregierungsorganisationen, Wirtschaftspartnern, Experten aus Wissenschaft und Forschung sowie internationalen Organisationen und Institutionen ausführlich hin. Die Einführung zu diesen ursprünglichen Abschnitten streicht heraus:

»The implementation of the Strategy can only be effective and efficient if all relevant societal actors agree upon and share its principal goals and values as basic orientations for their involvement and commitment; and if they are fully aware of their respective roles and willing to live up to their various responsibilities in contributing to the integration, participation and well-being of all people and to the social and economic development of the region's rapidly ageing societies."

Die insgesamt 23 Paragraphen zu diesem Thema wurden allerdings in den weiteren Textverhandlungen durch die Mitgliedsländer ersatzlos gestrichen. Die in Berlin verabschiedeten Dokumente enthalten zwar auch weiterhin entsprechende Hinweise, allerdings sind sie weniger exponiert und nicht in einem eigenen Passus nochmals gebündelt. Außerdem wird auf eine explizite Benennung von Wissenschafts- und Wirtschaftspartnern verzichtet. Vielmehr wird nur noch von »gesellschaftlichen Akteuren« oder dem »Privatsektor « gesprochen.

Es ist bedauerlich, dass die Mitgliedsländer von der ursprünglichen ausführlichen Benennung von Bündnispartnern abgekommen sind. Für eine enge Zusammenarbeit verschiedener Gruppierungen zu werben, wäre sicherlich eine gute Gelegenheit zur Intensivierung zukünftiger Kooperationen gewesen. Umso wichtiger ist es nun, sich auf nationaler Ebene für eine solche Zusammenarbeit einzusetzen. Außerdem sollten weitere Bündnispartner gewonnen werden. So fehlt beispielsweise in dem UNECEPapier die Benennung der Medien als weiterer bedeutsamer Kooperationspartner. Zwar wird in den Paragraphen 12,14, 18 und 19 auf die Bedeutung eines realistischen Altersbildes hingewiesen; inwieweit aber die Medien bei einer solchen Zielsetzung Hilfestellung leisten können und müssen, wird nicht vertieft. Es ist zu hoffen, dass auch in Zukunft Wissenschaft und Zivilgesellschaft eng eingebunden werden, wenn Regierungen sich über Maßnahmen innerhalb einer alternden Gesellschaft verständigen. Wünschenswert wäre darüber hinaus die Mobilisierung und Integration von maßgeblichen Wirtschafts- und Medienvertretern. Möglicherweise sind auch weitere Gruppierungen einzubeziehen (etwa Berufsverbände, Bildungsträger u. a.). Nur durch die Berücksichtigung verschiedener, ineinander greifender Zahnräder, die für die unterschiedlichen Koalitionäre stehen, kann das große gesellschaftliche Getriebe störungsfrei laufen, das zur Lösung sozialer Problemlagen beitragen soll.

Eine zukunftsfähige Sozialpolitik verlangt vor diesem Hintergrund ein »Bündnis für den demografischen Wandel« etwa nach dem Vorbild des Bündnisses für Arbeit, das führende Vertreter der verschiedenen Bereiche an einen Tisch bringt. Dabei sollten auch andere bestehende Instanzen genutzt werden. So hat die Bundesregierung einen Rat für nachhaltige Entwicklung berufen, dem führende Vertreter aus Wirtschaft, Gewerkschaften, Kirchen, Umweltverbänden und Wissenschaft angehören. Diese Ide- 
engeber könnten auch für das oben geforderte »Bündnis für den demografischen Wandel « als wichtige Berater fungieren. Gleichzeitig muss deutlich werden, was jeder Einzelne bewegen kann. Gesellschaftspolitische Partizipation ist dabei sowohl als Privileg wie auch als Verpflichtung für Bürgerinnen und Bürger zu verstehen.

\section{Auf berufliche Implikationen reagieren}

Die Auswirkungen des demografischen Wandels berühren die unterschiedlichsten Berufs- und Arbeitsfelder. Vor allem der Wissenstransfer zwischen Theorie und Praxis muss weiterhin verbessert werden. Die Einbeziehung unterschiedlicher Perspektiven, Erfahrungen und Schwerpunkte macht hierbei eine interdisziplinäre Zusammenarbeit besonders dringlich. Die Erfüllung dieser Forderung ist erfahrungsgemäß im Alltag nicht immer einfach. Ursache dafür ist oftmals eine einseitige Prägung, die eine interdisziplinäre Kooperation eher als Konkurrenz denn als Erweiterung des eigenen Fachgebietes erlebt. Aber auch Unterschiede im gesellschaftlichen Ansehen, Abweichungen im Verdienst sowie rigide Hierarchievorstellungen beeinträchtigen zuweilen die Zusammenarbeit zwischen einzelnen Berufsgruppen.

Will man dagegen ernsthaft die einzelnen Mosaiksteine aus Theorie und Praxis verschiedener Professionen zu einem gemeinsamen Bild integrieren, bedarf es einer gezielten Koordination der verschiedenen Vertreter ebenso wie einer engen Kooperation, die auf Partnerschaft und gegenseitiger Anerkennung beruht. Nur auf diesem Weg lassen sich die Erkenntnisse der verschiedenen Berufssparten gleichberechtigt integrieren. Wir benötigen dazu ein permeables - zwischen verschiedenen Disziplinen durchlässiges - Ausbildungswesen, das zu einem verstärkten Kontakt zwischen einzelnen Arbeitsfeldern führt. Bestehende Erkenntnisse müssen möglichst zeitnah, allgemein zugänglich und verständlich dokumentiert werden. Derart transparente Ergebnisse müssen sich auch der Realität stellen und nach fortlaufenden Überprüfungen weiter angepasst werden können.

Im Hinblick auf einen engen Theorie-Praxis-Transfer kommt insbesondere den Fachhochschulen zukünftig eine wachsende Bedeutung zu. Dazu ist jedoch ein Paradigmenwechsel unerlässlich. Das historische Ausmaß des demografischen Wandels findet an den Fachhochschulen im Rahmen einer generalistischen Ausbildung und eines integrierten Studiums noch keine flächendeckende Berücksichtigung. Zwar hat sich in den letzten Jahren durch den verstärkten Ausbau der Pflegewissenschaft ein stärkeres Selbstbewusstsein für die Pflege als eigenständige Disziplin herausgebildet. Jedoch bleibt das Thema Gesundheit und Alter noch immer ein eher randständiges Gebiet. Von den heute rund 160 Fachhochschulen verfügen zudem mehr als ein Drittel der Hochschulen über eigene Fachbereiche auf dem Gebiet Sozialwesen. Von wenigen Ausnahmen abgesehen, sind die Arbeitsgebiete Alter und Altern seit den 80er Jahren aber noch immer traditionell im Rahmen der Altenhilfe angelegt. Die multidimensionalen Auswirkungen des demografischen Wandels bleiben demgegenüber unterbelichtet. Neuorientierungen der Curricula und veränderte Akzente in der anwendungsbezogenen Forschung sind daher geboten. 
Eine solche Neubewertung erfordert die Schaffung neuer Lehrstühle und die inhaltliche Ergänzung bestehender Professuren. Der Wissenschafts- und Bildungsauftrag muss hierbei über die Beschreibung der sozial-kulturellen Wirklichkeit hinausgehen. Anzustreben ist zusätzlich ein Gestaltungsauftrag, der Veränderungen etwa im Berufsfeld der Sozialen Arbeit und im Gesundheitswesen antizipiert und berufspraktische Impulse gibt. Soziale und gesundheitliche Problemlagen werden durch den demografischen Wandel teilweise verschärft oder sind neu zu bewerten. Gleichzeitig ist durch das Altern der Gesellschaft mit völlig neuen Arbeitsfeldern zu rechnen. Die Fachhochschulen müssen auf diese Veränderungen auf der Grundlage wissenschaftlicher Methoden und Erkenntnisse rechtzeitig reagieren, die Studierenden auf diese Entwicklungen vorbereiten und entsprechende Kompetenzen vermitteln. Mit dem Ziel einer Reduktion sozialer Probleme und einer Optimierungsoffensive in den Bereichen Gesundheit und Pflege können die Fachhochschulen durch ihre enge Anbindung an die Berufspraxis einen wichtigen Beitrag zur Bewältigung des demografischen Wandels leisten. Im Sinne eines Seismographen vermögen die Fachhochschulen gerade auf aktuelle Veränderungen in den zugehörigen Praxisfeldern sensibel zu reagieren. Es bleibt zu hoffen, dass sich zunehmend mehr Fachhochschulen dieser neuen Anforderung stellen.

Ein Ausbau der Fachhochschulen in diesem Bereich ist nicht in Konkurrenz zu den Universitäten, sondern als komplementäre Maßnahme zu verstehen. Erfreulicherweise haben gerontologische Erkenntnisse in den letzten Jahren an verschiedenen Fachbereichen der Universitäten verstärkt Einzug gehalten. Angesichts der wachsenden Bedeutung dieses Themas verwundert jedoch nach wie vor die geringe Anzahl an gerontologisch orientierten Lehrstühlen und Instituten und die mangelnde Entwicklung fächerübergreifender Perspektiven in Forschung und Lehre.

\section{Solidarität unterstützen und ausbauen}

Eine Reihe von Studien hat in der Vergangenheit die enormen gegenseitigen Unterstützungsleistungen zwischen den Generationen in der Familie belegt. In breitem Umfang lassen sich beeindruckende freiwillige Finanzleistungen und immense immaterielle Formen der Unterstützung beobachten (vgl. Wagner, Motel, Spieß \& Wagner, 1996). Nach Befunden des Alters-Surveys erhalten 41 Prozent der 70- bis 85-Jährigen instrumentelle Unterstützung von außerhalb des Haushalts. Mit 21 Prozent rangieren dabei die Hilfeleistungen der eigenen Kinder weit vor denen bezahlter Helfer (6 Prozent) oder von Freunden (3 Prozent) (vgl. Kohli, Kühnemund, Motel \& Szydlik, 2000).

Während demnach die Familie einen Ort reger und individuell ausgehandelter Transferleistungen zwischen den Generationen definiert, sind die finanziellen Austauschbeziehungen außerhalb der Familie als Generationenvertrag gesetzlich fixiert. Im Sinne eines gerechten gesellschaftlichen Lastenausgleichs sind aber nicht allein intergenerationelle Unterstützungen geboten, sondern in zunehmendem Maße auch intragenerationelle Leistungen unumgänglich. Innerhalb der Gruppierung älterer Menschen sind die Einkommensunterschiede ähnlich stark ausgeprägt wie in anderen Altersgruppen. Im vierten Altenbericht (BMFSFJ, 2002, S. 59) heißt es dazu: 
»Die während des Erwerbslebens ungleichen Möglichkeiten der Einkommenserzielung und Vermögensanhäufung und des Erwerbs von Anwartschaften auf Alterseinkommen bestimmen weitgehend das im Alter verfügbare regelmäßige Einkommen. Der kumulative Effekt der sozialen Ungleichheit über den Lebensverlauf bedingt eine hohe Variabilität der Alterseinkommen, die durch die Ausgestaltung des Sozialversicherungs- und Steuerrechts nur wenig abgemildert wird. Auch die geringere Lebenserwartung der unteren Einkommensgruppen ändert an der Heterogenität der Einkommen im hohen Alter nur wenig."

Damit Altersarmut auch weiterhin ein marginales Problem in Deutschland bleibt, sind gesetzliche Maßnahmen erforderlich, die eine extreme Einkommensheterogenität unter älteren Menschen abmildern. Angesichts des wachsenden Anteils älterer Menschen sind zudem Entlastungen jüngerer Generationen durch sozial verträgliche Umverteilungen auch innerhalb der älteren Generation eine folgerichtige Konsequenz.

\section{Anpassungen des Bildungssystems vornehmen}

Welche Konsequenzen sind mit der Aussicht verbunden, alt $\mathrm{zu}$ werden und was bedeutet es, in einer Gesellschaft zu leben, in der sich in nur hundert Jahren das Zahlenverhältnis zwischen Jung und Alt vollständig umkehrt? Diese Fragen lassen sich nicht leicht beantworten. Jedoch leisten Angebote in der Aus-, Fort- und Weiterbildung an dieser Stelle wichtige Hilfestellungen. Um auf das individuelle und gesellschaftliche Altern vorzubereiten, sind Grundschulen, weiterführende Schulen, Fachhochschulen, Universitäten, Akademien, Institute, Bildungsträger und Arbeitgeber gleichermaßen gefordert. Ein bildungsübergreifender Ansatz verlangt in diesem Zusammenhang, dass die verschiedenen Lerninhalte aufeinander aufbauen können und sich gegenseitig ergänzen. Alterswissen, Altersstile und Altersbilder werden entscheidend von der individuellen Sozialisation geprägt. Bildungsträger sind Teil dieser Sozialisation. Ihre Verantwortung beginnt damit bereits bei lernenden Kindern. Auch in Studium und Ausbildung können im Rahmen der Erwachsenenbildung Fertigkeiten und Fähigkeiten erworben werden, die den Umgang mit dem eigenen und kollektiven Alterungsprozess erleichtern oder erschweren.

Das Postulat des lebenslangen Lernens macht an dieser Stelle deutlich, dass es für Bildungsangebote keine Altersgrenzen geben darf. Während die Geragogik die pädagogischen Bedingungen, Begleiterscheinungen und Folgen des Alterungsprozesses prüft, setzt sich die Gerontogogik mit der Praxis der helfenden Lebensbegleitung zur Optimierung von Alterungsprozessen auseinander. Die Entwicklungspsychologie der Lebensspanne überwindet die Fixierung auf eine bestimmte Lebensphase und bezieht sich auf den gesamten Lebenslauf. Den Hintergrund dafür bildet die Biografieforschung als Horizont individuell möglicher und notwendiger Bildung. Bildung im Alter kennzeichnet in diesem Sinne die reflexive Auseinandersetzung mit dem eigenen Werdegang und lotet die individuellen Möglichkeiten für ein Weiterlernen, Umlernen und Neulernen aus. Im Vordergrund der Bildungsangebote sollte eine Kompetenzorientierung stehen. Lernmöglichkeiten bieten unter dieser Prämisse die Voraussetzung zur Wiedererlangung oder Neugewinnung von Fähigkeiten und berücksichtigen sowohl 
biografische als auch institutionelle Barrieren. Das Recht auf lebenslanges Lernen impliziert auch im Arbeitsleben die Gewährleistung entsprechender Qualifizierungsangebote. Arbeitgeber und Arbeitnehmer müssen dazu noch in vielen Fällen destruktive Altersstereotype überwinden, die eine wesentliche Hürde bei der Bereitstellung, Akzeptanz und Nutzung von Fort- und Weiterbildungsangeboten am Arbeitsplatz darstellen.

Weitere strategische Überlegungen gehen dahin, die allgemein übliche Sequenz von Ausbildungszeit, Erwerbszeit und Freizeitphase in ihrer rigiden Reihenfolge zu durchbrechen (vgl. Bäcker \& Naegele, 1993). Die Forderung nach neuen Leitbildern für Bildung und Ausbildung über die gesamte Lebensspanne ist nicht neu. Niederfranke (2000, S. 301) weist in diesem Zusammenhang darauf hin, dass Bildung nicht allein als Qualifizierung und Tätigkeiten nicht allein als Erwerbsarbeit verstanden werden dürfen. Eine »querschnittliche Anschubfunktion« der Altenpolitik hat sich dann aus ihrer Sicht im positiven Sinne des Wortes »selbst überholt«, wenn dieses Verständnis erreicht ist. Altenpolitik kann hier als innovativer Impulsgeber wirken, um das entsprechende Rüstzeug für einen konstruktiven Umgang mit den sich verändernden Anforderungen in einer alternden Gesellschaft bereit zu stellen.

\section{Gesamtgesellschaftliche Maßnahmen entwickeln}

Hinter dem Begriff »ältere Menschen« verbirgt sich eine Vielzahl von Gruppierungen mit verschiedenen Interessen, Bedürfnissen, Problemlagen und Kapazitäten. Politische Entscheidungen können außerordentlich unterschiedliche Auswirkungen auf diese Subgruppen haben. Auch die klassische Altenpolitik ist daher gut beraten, entsprechende Maßnahmen für die verschiedenen Zielgruppen genauer zu beleuchten. Anhand einiger Beispiele sollen im Folgenden einige dieser Zielgruppen benannt werden.

Obwohl die Festlegung von Altersgrenzen durch das kalendarische Alter keine Vorhersage individueller Altersverläufe erlaubt, lassen sich derartige Klassifikationen für die Beurteilung von Gruppen wirkungsvoll nutzen. Auch in Deutschland setzt sich in diesem Zusammenhang zunehmend die Unterteilung in das dritte und vierte Lebensalter durch. Das dritte Lebensalter umfasst in der Regel die »jungen Alten« von 60 bis 79 Jahren. Das vierte Lebensalter bezieht ältere Menschen ab 80 Jahren ein. Aufgrund des rasanten Anstiegs Hochaltriger ist allerdings zu erwarten, dass sich ähnlich wie in den USA die Altersgrenze des vierten Lebensalters um mindestens fünf weitere Jahre nach hinten verschiebt (vgl. Wahl \& Rott, 2002). Während die jungen Alten in der Regel mit den Themen Partizipation und Produktivität verknüpft werden, finden sich bei den Hochaltrigen zumeist negativere Zuschreibungen, die auf das wachsende Risiko der Pflegebedürftigkeit und der abnehmenden Autonomie verweisen. Für altenpolitische Initiativen macht diese Unterteilung in mindestens zwei unterschiedliche Generationen die Notwendigkeit neuer und passgenauer Konzepte klar ersichtlich.

Gleichzeitig ist eine paradoxe Entwicklung zu beobachten, die trotz steigender Lebenserwartung und zunehmender Zahl gesunder und aktiver Lebensjahre eine immer frühzeitigere Kategorisierung als »alt« mit sich bringt. Dieser Prozess vollzieht 
sich besonders ausgeprägt auf dem Arbeitsmarkt. Aufgrund der in Deutschland bestehenden Frühverrentungstrends und wegen der relativ geringen Erwerbsbeteiligung der ab 55-Jährigen werden Arbeitnehmer und Arbeitnehmerinnen zunehmend früher als alt bezeichnet. Altenpolitik beginnt insofern nicht erst nach dem klassischen Berentungsalter, sondern muss sich auch mit anderen Definitionen und Auswirkungen von Alterskategorisierungen befassen.

Die Lebenserwartung von Frauen liegt höher als diejenige der Männer. Geschlechtsspezifische Unterschiede bezüglich der Mortalität sind weltweit anzutreffen, und es existieren unterschiedliche Theorien, die dieses Phänomen zu erklären versuchen. In Deutschland beträgt die weitere Lebenserwartung für die Altersgruppe von 60 Jahren für Frauen 23,3 Jahre und für Männer 19,0 Jahre. Im Alter von 80 Jahren liegt sie für Frauen bei 8,4 Jahren und für Männer bei 6,9 Jahren (Statistisches Bundesamt, 2001). Gleichzeitig sind Frauen im Alter aber im Vergleich zum männlichen Geschlecht auch kränker, leben häufiger allein und verfügen über ein geringeres Einkommen (vgl. BMFSFJ, 2002). Diese Aufzählung macht deutlich, dass der Gender-Aspekt für die Altenpolitik eine ganz wesentliche Rolle spielt.

Die Daten der Schwerbehindertenstatistik vom 31. Dezember 1999 belegen zudem eine starke Alterslastigkeit von Behinderungen. Drei Viertel der Schwerbehinderten sind nach den Ergebnissen der Versorgungsämter 55 Jahre oder älter. Der Anteil der unter 25-Jährigen fällt demgegenüber mit nur 3,9 Prozent relativ gering aus. Infolge des Europäischen Jahres der Menschen mit Behinderung hat die Europäische Kommission am 30. Oktober 2003 einen Aktionsplan zur Förderung der Chancengleichheit von Menschen mit Behinderungen in einem erweiterten Europa vorgelegt. Bis zum Jahr 2010 wird hierbei ein verstärkter Erfahrungsaustausch und Datentransfer zwischen den EU-Partnern gefordert. Es bleibt abzuwarten, ob auf diesem Weg auch das Thema Alter und Behinderung stärker in den Fokus der Aufmerksamkeit gerät.

Die Liste weiterer möglicher Zielgruppen ist lang. Die Notwendigkeit eines gesamtgesellschaftlichen Ansatzes unterstreicht nochmals die Notwendigkeit eines differenzierten sozialpolitischen Vorgehens.

\section{Ausblick}

Bereits die skizzenhaften Ausführungen in diesem Beitrag belegen, dass die demografische Entwicklung zum Dreh- und Angelpunkt unserer Gesellschaft wird. Altenpolitische Maßnahmen müssen deutlich an Profil und Bedeutung gewinnen, wenn sie den steigenden Anforderungen gewachsen sein wollen. Dazu braucht es vielfache Unterstützung von unterschiedlichen Seiten. Zudem ist eine grundsätzliche Bereitschaft nötig, sich insbesondere mit unbequemen sozialpolitischen Fragen auseinanderzusetzen. Ob der demografische Wandel in erster Linie mit Chancen oder mit Problemen verbunden ist, hängt wesentlich von der Umsetzung eines ernsthaften und glaubwürdigen Querschnittsansatzes ab. Ohne bereichsübergreifendes, konsequentes Handeln bleibt die geforderte Querschnittspolitik hingegen eine leere Formel. Die negativen Folgen des damit verbundenen politischen Stillstands wären bereits heute absehbar und die daraus resultierenden sozialen und wirtschaftlichen Kosten enorm. 
Der aktuelle internationale Aufschwung für das Thema Alter muss daher gerade auf nationaler Ebene als zusätzliche argumentative Stützung eines konsequenten Querschnittsansatzes auch in Deutschland genutzt werden. Tatsächlich wurden von deutscher Seite in dieser Hinsicht klare Absichtserklärungen gegeben. In den Koalitionsvereinbarungen der Bundesregierung (Koalitionsvertrag, 2002) ist auf S. 58 in einem eigenen Absatz zur Politik für ältere Menschen nachzulesen:

»Auf der Basis des im April 2002 in Madrid verabschiedeten 2. Weltaltenplans und der im September in Berlin von der Wirtschaftskommission der Vereinten Nationen für Europa beschlossenen regionalen Umsetzungsstrategie werden wir für Deutschland einen Nationen Aktionsplan zur Bewältigung der demografischen Herausforderungen erarbeiten und umsetzen «.

Es bleibt zu hoffen, dass dieser Ankündigung eines nationalen Aktionsplans auch bald sichtbare Ergebnisse folgen und dass dieser Prozess eine breite gesellschaftliche Unterstützung erfährt. Die Bemühungen der Vereinten Nationen unterstreichen, dass die Politik die Brisanz des demografischen Wandels richtig erkannt hat. Nun muss es darum gehen, aus dieser Erkenntnis die richtigen Schlüsse für ein schnelles und effizientes Handeln zu ziehen. Um mit Senecas rund 2400 Jahre altem Grundsatz zu schlieBen:

»Es ist nicht wenig Zeit, was wir haben, sondern es ist viel, was wir nicht nützen.«

\section{Literatur}

Bäcker, G. \& Naegele, G. (1993). Alternde Gesellschaft und Erwerbstätigkeit. Modelle zum Übergang vom Erwerbsleben in den Ruhestand. Köln: Bund-Verlag.

Bosch, E. M. (1990). Altersbilder in den bundesdeutschen Medien. In: G. A. Straka, T. Fabian \& J. Will (Hrsg.), Aktive Mediennutzung im Alter, 77-91. Heidelberg: Asanger.

BMFSFJ - Bundesministerium für Familie, Senioren, Frauen und Jugend (2002). Risiken, Lebensqualität und Versorgung Hochaltriger - unter Berücksichtigung demenzieller Erkrankungen. Vierter Bericht zur Lage der älteren Generation in der Bundesrepublik Deutschland. Berlin.

Büro gegen Altersdiskriminierung (2002). Lebensalter und Diskriminierung. Dokumentation des ersten bundesweiten Beschwerdetages zum Thema Altersdiskriminierung. Köln: Selbstverlag.

Deutscher Bundestag (2002). Enquête-Kommission Demographischer Wandel. Herausforderungen unserer älter werdenden Gesellschaft an den Einzelnen und die Politik. Paderborn: Media-Print.

Dieck, M. (1991). Altenpolitik. In: W. D. Oswald, W. M. Hermann, S. Kanowski, U. Lehr \& H. Thomae (Hrsg.), Gerontologie. 23-37. Stuttgart: Kohlhammer.

Dörner, D., Kreuzig, H., Reither, F. \& Stäudel, T. (Hrsg.) (1995). Lohausen - Vom Umgang mit Unbestimmtheit und Komplexität. Bern: Hans Huber.

EU-Nachrichten (2004). Grünbuch Gleichstellung - Vorurteile abbauen. Nr. 23, 17. 6. 2004, S. 5.

Gronemeyer, R. (1991). Die Entfernung vom Wolfsrudel. Über den drohenden Krieg der Jungen gegen die Alten. Frankfurt a. M.: Fischer. 
Koalitionsvertrag (2002). Erneuerung - Gerechtigkeit - Nachhaltigkeit: Für ein wirtschaftlich starkes, soziales und ökologisches Deutschland. Für eine lebendige Demokratie.

Kohli, M., Kühnemund, H., Motel, A. \& Szydlik, M. (2000). Grunddaten zur Lebenssituation der 40- bis 85-jährigen deutschen Bevölkerung. Ergebnisse des Alters-Survey. Berlin: WeiBensee.

Mohl, H. (1993). Die Altersexplosion. Droht uns ein Krieg der Generationen? Stuttgart: Kreuz Verlag.

Niederfranke, A. (2000). Altenpolitik. In: H.-W. Wahl, \& C. Tesch-Römer (Hrsg.), Angewandte Gerontologie in Schlüsselbegriffen, 386-392. Stuttgart: Kohlhammer.

Pohlmann, S. (2003). Altern gestalten - Konstruktive Antworten auf Fragen der Bevölkerungsentwicklung. Regensburg: Transfer Verlag.

Presse- und Informationsdienst der Bundesregierung (1999). Vertrag von Amsterdam - Texte des EU-Vertrages und des EG-Vertrages mit den deutschen Begleitgesetzen. Bonn: Europa Union.

Presse- und Informationsdienst der Bundesregierung (2001). Rede von Staatsminister Bury zur abschließenden Beratung des Antrags der Koalitionsfraktionen »Nationale Nachhaltigkeitsstrategie«. Veröffentlicht am 19. Oktober 2001. www.bundesregierung.de.

Schüller, H. (1995). Die Alterslüge. Für einen neuen Generationenvertrag. Berlin: Rowohlt.

Statistisches Bundesamt (2001). Abgekürzte Sterbetafel 1997/99. In: Statistisches Bundesamt (Hrsg.). Fachserie 1: Bevölkerung und Erwerbstätigkeit. Reihe 1: Gebiet und Bevölkerung. Wiesbaden, 246-247.

Thomae, H. (1983). Alternsstile und Altersschicksale. Bern: Hans Huber.

UNECE - United Nations Economic Commission for Europe (2002). Regional Implementation of the International Plan of Action on Ageing. Geneva: UNECE.

UNECE - United Nations Economic Commission for Europe (2002b). ECE/AC.23/2002/2/ Rev.3 vom 10. Juni 2002.

UNFPA (2001). United Nations Population Fund. Deutsche Stiftung Weltbevölkerung. Weltbevölkerungsbericht 2001: Bevölkerung und Umwelt. Stuttgart: Balance.

United Nations (1982). International Plan of Action on Ageing. New York: United Nations.

United Nations (2002). Madrid International Plan of Action on Ageing. New York: United Nations.

United Nations (2002b). World Population Ageing 1950-2050. Population Division, DESA (ST/ESA/SER.A/207). New York: United Nations.

United Nations (2002b) A world fit for children A/S-27/19/Rev.1. New York: United Nations.

Wagner, G., Motel, A., Spieß, K. \& Wagner, M. (1996). Wirtschaftliche Lage und wirtschaftliches Handeln alter Menschen., In: K. U. Mayer \& P. B. Baltes (Hrsg.), Die Berliner Altersstudie, 277-299. Berlin: Akademie Verlag.

Wahl, H.-W. \& Rott, C. (2002). Konzepte und Definitionen der Hochaltrigkeit. In: Das hohe Alter - Konzepte, Forschungsfelder, Lebensqualität. Hannover: Vincentz. 